\title{
Shape-from-silhouettes algorithm with built-in occlusion detection and removal
}

\author{
Maarten Slembrouck ${ }^{1}$, Dimitri Van Cauwelaert ${ }^{1}$, Peter Veelaert ${ }^{1}$ and Wilfried Philips ${ }^{12}$ \\ ${ }^{1}$ TELIN dept. IPI/iMinds, Ghent University, Valentin Vaerwyckweg 1, Ghent, Belgium \\ ${ }^{2}$ TELIN dept. IPI/iMinds, Ghent University, Sint-Pietersnieuwstraat 41, 9000, Ghent, Belgium \\ maarten.slembrouck@ugent.be
}

\begin{abstract}
Keywords: $\quad$ Multi-camera, Occlusion, Visual hull, 3D modelling
Abstract: Occlusion and inferior foreground/background segmentation still poses a big problem to 3D reconstruction from a set of images in a multi-camera system because it has a destructive nature on the reconstruction if one or more of the cameras do not see the object properly. We propose a method to obtain a 3D reconstruction which takes into account the possibility of occlusion by combining the information of all cameras in the multicamera setup. The proposed algorithm tries to find a consensus of geometrical predicates that most cameras can agree on. The results show a performance with an average error lower than $2 \mathrm{~cm}$ on the centroid of a person in case of perfect input silhouettes. We also show that tracking results are significantly improved in a room with a lot of occlusion.
\end{abstract}

\section{INTRODUCTION}

Shape-from-silhouette algorithms are widely used to reconstruct a 3D object from a set of images and perform very well in case of artificial circumstances. However, in the real world occlusions are common to occur. Occlusion has a destructive nature on traditional shape-from-silhouettes algorithms because it carves away a part of the volume that should belong to the object. A person may for instance be partially occluded from a camera viewpoint, resulting in only half of the person becoming foreground, which is not an immediate problem, but since the location of occlusion at that point is unknown, 3D reconstruction will become very hard for traditional shape-from-silhouettes algorithms (visual hull) [Laurentini, 1994, Laurentini, 1997, Laurentini, 1999]. In [Guan et al., 2006] this problem is tackled by trying to find the occluded pixels in the camera view by using prior knowledge about occluders. Occluders most often give birth to straight lines in the foreground/background segmentation. When this is not the case however, the algorithm will fail to recover occluded parts.

Ober-Gecks et al.also uses a shapes-fromsilhouettes algorithm in the field of robotics to reconstruct persons in an indoor environment for safety reasons [Ober-Gecks et al., 2014]. After their implementation of the visual hull concept, temporal filter- ing is used to address detection failures of the cameras. They also handle occlusion in each processing step by integrating context knowledge in their tracking steps. In case of dynamic occlusion due to the displacement of an object such as a chair this context should be altered which is not the case in this algorithm.

Stengel et al. presents an efficient reconstruction which exploits per-voxel data parallelism to efficiently perform voxel-to-silhouette tests and handles occlusion using a predefined modelling of moving object such as robots to evaluate whether there is occlusion or not [Stengel et al., 2012]. This means that the area has to be well defined and cannot be changed easily. For example if the robots needs to move to another corner of the working space this information needs to be manually adapted into the system.

The automatic occlusion detection algorithm described in [Slembrouck et al., 2014] creates an occlusion map based on a voting mechanism taking into account the position of the cameras and the number of cameras that agree certain voxels are occluded while a person walks around in the room. This algorithm could potentially be refined in case of partial occlusion as the proposed algorithm suffers from inaccuracies at the border of occluding objects, because only a handful of voxels which are occluded are detected in that phase.

On the other hand foreground/background seg- 
mentation algorithms are improving every year. However, in real world environments there are still some major issues. For example when a person wears a colour that is similar to the background, a lot of the presumed foreground is not detected. The CDnet 2014 data set [Wang et al., 2014] shows there is still room for improvement on foreground/background segmentation algorithms. The best algorithm still stays below $75 \%$ F-measure. Foreground/background segmentation is not seldom a first step in a much broader application. Therefore it is crucial this step delivers reliable results.

In this paper we tackle the problem of occlusion and bad foreground/background segmentation by combining information from multiple cameras which are observing the same scene from different viewpoints. For instance when a person stands in front of a wall which has a similar colour, this will be badly detected from a camera that sees the person in front of the wall, but the foreground segmentation will be much better for viewpoints that see the person in front of another background.

Our algorithm is able to reconstruct a complete person even in the presence of occlusion where some bodyparts are partially or fully occluded. Therefore we use a consensus of geometrical predicates.

The contribution is twofold. The first is a way to determine occluded parts of the scene for each camera which makes it possible to remove occlusion and the second is an effective way to improve the foreground/background segmentation by combining multiple camera viewpoints, even tough our algorithm was not designed for this.

In section 2 we take a look at the problem at hand while in section 3 we will explain our algorithm in detail. Section 4 will show promising results both for simulations and for real data.

\section{Occlusion problem}

We propose a 3D reconstruction algorithm which distinguishes from a traditional shape-fromsilhouettes algorithm in the sense that the result does not equal the enclosed volume of the backprojected silhouettes from each camera. In case of occlusion or inferior foreground/background segmentation the resulting 3D object is severely deformed using this approach. In case of full occlusion in at least one camera view, no 3D shape is detected at all. With our algorithm we will propose a way to obtain an acceptable $3 \mathrm{D}$ reconstruction even in the presence of severe occlusion in multiple camera views without any knowledge of the scene.

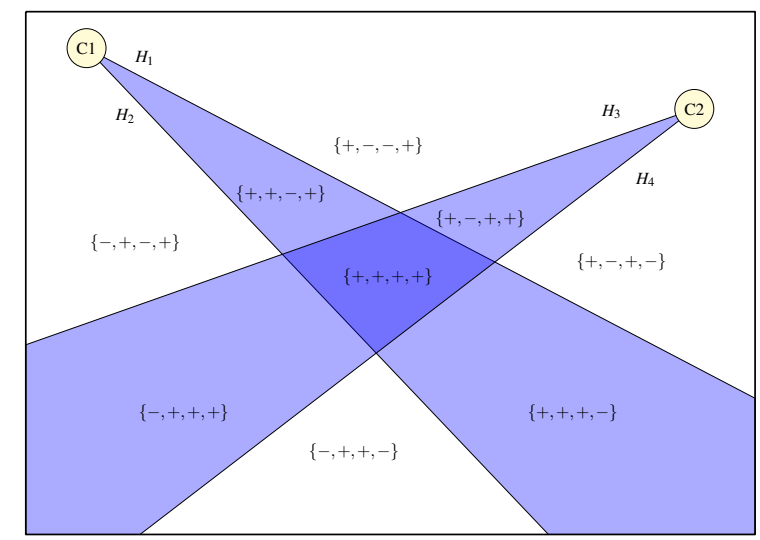

Figure 1: Example of the construction of cells with two cameras, which introduces the sign vector: $\left\{S_{1}, S_{2}, S_{3}, S_{4}\right\}$ where each $S_{j}$ represents + or - depending on which side of the lines $H_{j}$ they occur.

The input of the algorithm is the same as that of traditional shape-from-silhouettes algorithms: a calibrated camera environment and a synchronized set of silhouette images.

\subsection{Subdividing the space in cells}

In this section we will explain the concept behind our algorithm in two-dimensional space and for a convex object. Extension to a three-dimensional space and a non-convex object is straightforward. We use the same notation as in chapter 6 about oriented matroids of [Toth et al., 2004].

For a real matrix $X:=\left(x_{1}, \ldots, x_{n}\right) \in\left(\mathbb{R}^{2}\right)^{n}$, we consider the system of hyperplanes $\mathcal{H}_{x}:=$ $\left(H_{1}, \ldots, H_{n}\right)$ with

$$
H_{l}:=\left\{\gamma \in \mathbb{R}^{2}: \gamma^{T} x_{l}=0\right\} .
$$

Let $\gamma=\left(\alpha_{1}, \alpha_{2}, \alpha_{3}\right)^{T}$ and $x_{l}=\left(x_{l, 1}, x_{l, 2}, 1\right)^{T}$, then each vector $x_{l}$ induces an orientation on $H_{i}$ by defining

$$
H_{l}^{+}:=\left\{\gamma \in \mathbb{R}^{2}: \gamma^{T} x_{l}>0\right\},
$$

to be the positive side of $H_{l}$, while $H_{l}^{-}$is analogously defined as the negative side of $H_{l}$.

The projection of a convex $2 \mathrm{D}$ object on the sensor of the cameras is a line segment. In figure 1 we see that every pair of half planes $H_{i}$ and $H_{j}$ defines a double cone where $H_{i}^{+} \cap H_{j}^{+}$is represented as the blue area. They divide the space in cells. Every cell $C$ can be described by a unique sign vector. The signs are based on the halfplanes that divide the space $\left(H_{1}\right.$, $H_{2}, H_{3}$ and $H_{4}$ in this example). The sign vector indicates if a cell lies on the positive side of $H_{i}$ with + or the negative side with - . A sign vector has the form

$$
\left\{S_{1}, S_{2}, \ldots, S_{M}\right\},
$$


where $S_{i} \in\{+,-\}$. In the case every camera has one line segment, $M:=2 N$ with $N$ the number of cameras.

Using the halfplanes we are able to define a cell as the intersection of halfplanes, for instance

$$
\mathcal{C}=H_{1}^{+} \cap H_{2}^{+} \cap H_{3}^{+} \cap H_{4}^{+}
$$

is the dark blue region in figure 1, also known as the visual hull.

Note that the length of the sign vector depends on the number of line segments on the sensors of each camera. Each interval on the line sensor introduces two extra positions in the sign vector. For simplicity, we assume one interval per camera sensor, but the theory can be extended for higher complexity.

In that case, the traditional shape-from-silhouettes algorithm only retains the cell where the sign vector has pluses at all its positions. However, in case of occlusion this is not enough. In figure 2 for instance the orange object $\Omega$ is partially blocking the view of camera 1 . The traditional shape-from-silhouette algorithms will lose a big part of the object in this case. Only cell A will be kept. Our algorithm allows to add cells to the traditional result, we call them augmenting cells. These cells will contain minuses which practically means that the half planes corresponding to the minus will be ignored so cell $\mathrm{B}$ can be added.

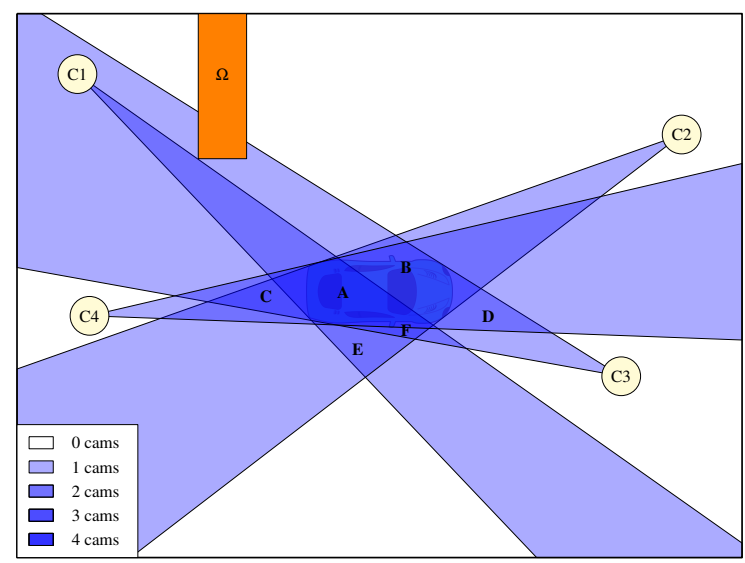

Figure 2: Subdividing the space in cells with occlusion present (occluder $\Omega$ is indicated in orange). Visually we see that the desired solutions is the union of cell $\mathrm{A}$ and $\mathrm{B}$ whereas cell C, D, E, F and all non-indicated cells should be rejected.

\subsection{Definitions}

In this section we extend our explanation to threedimensional space because our algorithm works on $2 \mathrm{D}$ images which are a projection of a $3 \mathrm{D}$ scene. This implicates that the line segments become silhouettes on the sensor, but the theory about sign vectors still holds, although the number of halfplanes increases significantly. In three-dimensional space the cells are only convex when the silhouettes are convex, but as the property of convexity is not used, this does not have serious implications.

In order to explain how our algorithm works, we define the following symbols:

$N \quad$ total number of cameras in the multi-camera setup

$s_{i} \quad$ union of a finite number of filled contours on the sensor of camera $i$, which corresponds to the projection of the object(s) we like to reconstruct

$\mathcal{H}$ traditional visual hull shape, the cell with all pluses as sign vector

$C^{k} \quad$ cell with index $k$ which is uniquely defined by a sign vector

We also define some operators:

$\Pi_{i}(\mathcal{A}) \quad$ projection of a convex shape $\mathcal{A}$ on the sensor of camera $i$

$\Pi_{i}^{-1}\left(b_{i}\right) \quad$ reprojection of the silhouette $b_{i}$ from camera $i$

$\mu\left(s_{i}\right) \quad$ the size of a silhouette on the camera sensor

With these operators, we define some additional symbols as shorthands:

$$
\begin{aligned}
h_{i} & :=\Pi_{i}(\mathcal{H}) \\
c_{i}^{k} & :=\Pi_{i}\left(C^{k}\right)
\end{aligned}
$$

These notations allow us to write the traditional visual hull concept in a single formula

$$
\mathcal{H}:=\bigcap_{i=1}^{N} \Pi^{-1}\left(s_{i}\right) .
$$

This formula expresses the concept of the shapefrom-silhouettes where the shape is the volume enclosed by the backprojection of all silhouettes.

We define the camera consistency $\beta_{\text {consist }}\left(C^{k}\right)$ of a cell $C^{k}$ as the number of cameras where the projection of this cell lies inside the silhouette,

$$
\beta_{\text {consist }}\left(C^{k}\right)=\sum_{i: c_{i}^{k} \subseteq s_{i}}^{N} 1 .
$$

The camera consistency set $A_{\text {consist_set }}$ of a cell $C^{k}$ is defined as the index set of camera indices where the projection of the cell lies inside the silhouette,

$$
A_{\text {consist_set }}\left(C^{k}\right)=\left\{i: c_{i} \subseteq s_{i}\right\} .
$$


The covering fraction of a cell on camera $i$ is the fraction of $c_{i}^{k}$ that lies inside the silhouette $s_{i}$, we express this with the following formula:

$$
x_{c o v, i}\left(C^{k}\right):=\frac{\mu\left(c_{i}^{k} \cap s_{i}\right)}{\mu\left(c_{i}^{k}\right)} .
$$

A cell $C^{k}$ is augmenting for camera $i$ if the predicate $P_{\text {aug }, i}\left(C^{k}\right)$ holds true:

$$
P_{\mathrm{aug}, i}\left(C^{k}\right):=\left(\left(c_{i}^{k} \subseteq s_{i}\right) \wedge\left(c_{i}^{k} \not h_{i}\right)\right) .
$$

It means that the cell is not a part of the projection of $\mathcal{H}$, but its projection lies inside the silhouette $s_{i}$.

In section 3.3 we will explain the criteria that decide if a cell is augmenting in three-dimensional space. Therefore we define the predicate $P_{\text {aug }}\left(C^{k}\right)$ which holds true when these criteria for cell $C^{k}$ are met. Note that this is different from equation 11 . $P_{\text {aug }}\left(C^{k}\right)$ is a decision based on all camera evidence, not just the projection on one camera.

A collection of cells is covering for camera $i$ if the projection of the union of the visual hull and all the extra added cells cover the whole silhouette,

$$
s_{i} \subseteq\left(h_{i} \cup\left(\bigcup_{k \in \Phi} c_{i}^{k}\right)\right),
$$

where $\Phi \subset K$ and $K$ is the collection of all cells

\section{Proposed algorithm}

The aim is to find the set of cells that is as small as possible, but does explain the silhouettes in the best possible way. The implementation operates in voxel space because the number of intersections in threedimensional space scales very bad. The disadvantage of using voxels is that we lose precision. For instance, small cells might not be detected, but it significantly improves runtime and small cells have little influence on the precision of the result. All silhouettes and projections are handled as pixels since the input images are also constructed with pixels.

We distinguish two major steps in our algorithm:

1. Calculation of the cells

2. Determination of the augmenting cells

\subsection{Calculation of the cells}

A cell is a set of voxels where each voxel has the same camera consistency set (equation 9 can also be applied on voxels) and where all voxels which belong to the set are connected with each other. Our approach to cluster the voxels in the correct cells works as follows. First, we loop over all voxels and determine for which cameras the projection of that voxel $(v)$ is a subset of the silhouette: $\Pi_{i}(v) \subseteq s_{i}$. Secondly, we pick a voxel and grow in all directions over the voxels which have the same camera consistency set. This process automatically halts when all voxels from the same cell are found. All these voxels are clustered together and stored. We also store the camera consistency $\beta_{\text {consist }}\left(C^{k}\right)$ for this cell (as we will also need that later on). The voxels that are clustered, are removed from the search space since each voxel belongs to one cell only. We repeat the clustering process until all voxels are clustered.

\subsection{Determination of the augmenting cells}

Our algorithm itself operates on the cells (clusters of voxels) we determined in the previous step. Let $T^{j}$ be the set of voxels which represents the 3D shape of all augmenting cells and the visual hull, this shape is updated after all cells with the same camera consistency are evaluated which means $j \in 0,1, \ldots, N$. It does not make much sense to include cells with very low camera consistency because they are usually very big and have little chance to belong to the actual object. The cells that are consistent for all cameras $\left(\beta_{\text {consist }}\left(C^{k}\right)=N\right)$ form the starting point of the algorithm. Therefore the current solution is equal to the result of the traditional visual hull algorithm:

$$
T^{0}=\mathcal{H}
$$

We chose to evaluate the cells in descending order of camera consistency because these cells have a major chance to be augmenting than cells with lower camera consistency. Once all cells with the same camera consistency are evaluated, we update the 3D shape $T^{j}$ by adding all augmenting cells. We define $K$ as $K:=\left\{k:\left(\beta_{\text {consist }}=N-j\right) \wedge P_{\text {aug }}\left(C^{k}\right)\right\}$ in order to update $T^{j-1}$ as

$$
T^{j}=T^{j-1} \cup\left(\bigcup_{k \in K} c^{k}\right) .
$$

Note that it is possible to add occluded parts using this approach because cells, that project within the silhouette in other cameras than the occluded camera, will add the cell to the shape.

\subsection{Criteria}

In this section we will discuss different criteria to determine whether a cell is augmenting or not. A significant measure is the covering fraction of a cell $C^{k}$ 
(equation 10). This fraction between 0 and 1 indicates the overlap of the projection of $C^{k}$ on camera $i$ and its filled silhouette $s_{i}$.

We distinguish three different fraction after projecting a cell $C^{k}$ on a camera:

- $x_{\text {non_expl }}$ : fraction that is part of the silhouette, but was not already explained by the solution $T^{j-1}$

$$
x_{\text {non_expl }}:=\frac{\mu\left(\left(c_{i}^{k} \cap s_{i}\right) \backslash \Pi_{i}\left(T^{j-1}\right)\right)}{\mu\left(c_{i}^{k}\right)}
$$

- $x_{\text {expl }}$ : fraction that is part of the silhouette which was already explained by another cell

$$
x_{\text {expl }}:=\frac{\mu\left(c_{i}^{k} \cap \Pi_{i}\left(T^{j-1}\right)\right)}{\mu\left(c_{i}^{k}\right)}
$$

- $x_{\text {extra }}$ : fraction that is not part of the silhouette and would add extra to the projection (only interesting if that part is occluded)

$$
x_{\text {extra }}:=\frac{\mu\left(c_{i}^{k} \backslash s_{i}\right)}{\mu\left(c_{i}^{k}\right)}
$$

These three parameters are used to determine whether a cell is augmenting or not. In the results section we discuss the value of the silhouette covering fractions, which will be called using the following convention:

$$
T_{\text {cov }}:=\text { silhouette covering threshold. }
$$

We define a predicate $P_{\text {cov,i }}\left(C^{k}\right)$ that indicates whether or not the silhouette is covered by a cell:

$$
P_{\mathrm{cov}, \mathrm{i}}\left(C^{k}\right)=\left(x_{\mathrm{cov}, i}\left(C^{k}\right)>T_{\mathrm{cov}}\right) .
$$

A cell is augmenting when there is a fraction of its projection on the camera which is not already explained by the current solution $x_{\text {non_expl }}>0$. However, for practical reasons due to imperfect calibration of the cameras and errors in the silhouettes, we need to add some more restrictions. We found that when the sum of the fraction that was not yet explained and the fraction that was already explained was smaller than the fraction that added extra, the cell was very unlikely to belong to the solution. On the other hand, when the extra added part was smaller, it is very likely that the cell contributes to the solution. Also when the overlapping area between the silhouette $s_{i}$ and the projection of the cell is high, the cell should be added to the solution. This reasoning gave us a criteria whether a cell is augmenting for a camera or not:

$$
\left(x_{\text {non_expl }}>0 \wedge x_{\text {non_expl }}+x_{\text {expl }}>x_{\text {extra }}\right) \vee P_{\text {cov }, i}\left(C^{k}\right)
$$

The silhouette covering part is added because in some cases we noticed that due to imperfect camera calibration the fractions where not sufficient to obtain the correct result.

The number of cameras that agree to add cell $C^{k}$ is equal to the number of cameras that meet the criteria in equation 20. If this number is higher than or equal to the camera consistency of that cell, we call the cell augmenting and we add it to the $3 \mathrm{D}$ shape.

\section{Results}

\subsection{Camera setup}

In this section we will discuss the results of our algorithm. For both the simulation and the real world test setup we use the same calibration to provide a fair comparison. The camera setup has seven cameras in an 8 by 4 meters area. All cameras are mounted a little over 3 meter on a truss construction. All cameras are type Allied Vision Technologies Manta G-046C [Allied Vision Technologies, ] cameras.

Figure 3 shows the xy-plane of our test setup. The $\mathrm{x}$-axis is horizontal (positive to the right) whereas the $y$-axis is vertical (positive to the bottom). The Vshaped occluder (in gray) is 2.12 metres heigh and 4 centimetres tick. The black squares are used as reference points for our measurements. There are 12 of these reference points in total. The positions of these points can be found in table $1^{1}$.

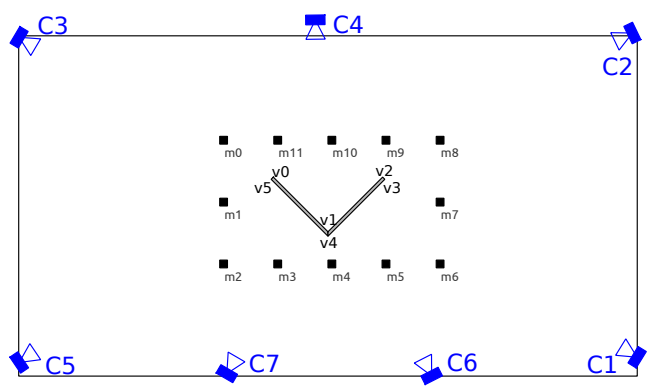

Figure 3: The test setup in our multi-camera environment. A total of seven cameras is used with a V-shaped occluder in the middle of the room (gray). The black squares m0-m11 are 12 reference points where we will evaluate our algorithm.

\footnotetext{
${ }^{1}$ All the material for the experiments can be found on our website.

http://telin.ugent.be/ mslembro/?q=node/18
} 


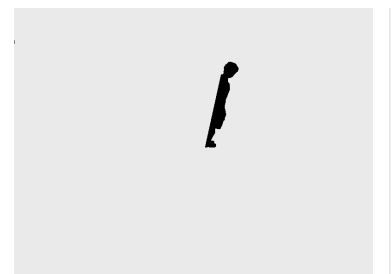

(a) mask of Cam 1

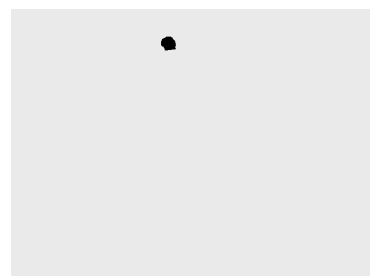

(e) mask of Cam 5

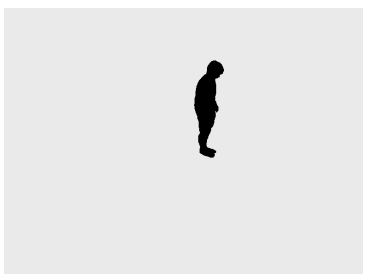

(b) mask of Cam 2

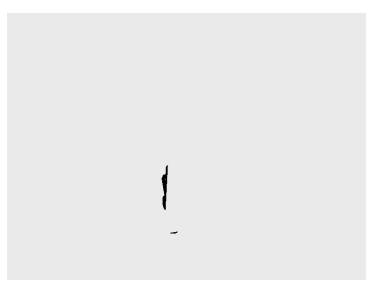

(f) mask of Cam 6

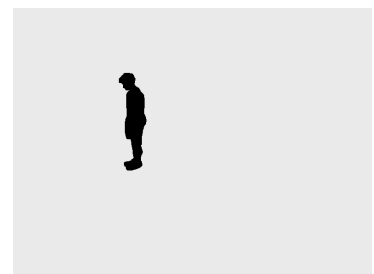

(c) mask of Cam 3

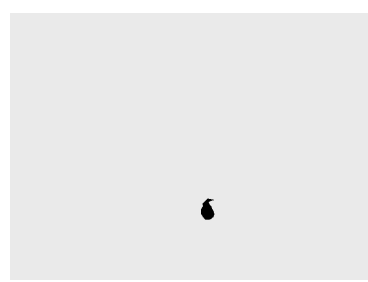

(g) mask of Cam 7

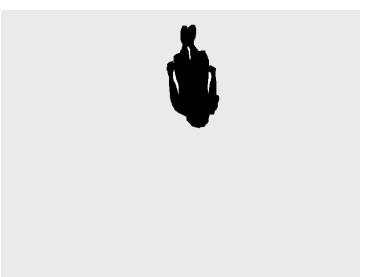

(d) mask of Cam 4

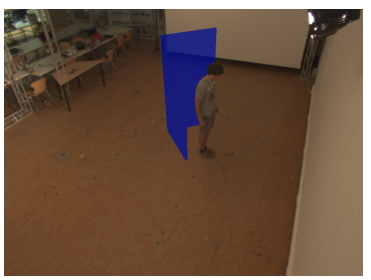

(h) Input of Cam 2

Figure 4: The foreground/background masks of the simulated camera setup. Cam 1, Cam 5, Cam 6 and Cam 7 are clearly occluded due to the wooden V-shape in the scene. In figure (h) we show the actual input image with the simulated V-shaped occluder present. The 3D reconstruction of these images can be found in figure 5 .

\begin{tabular}{|l|r|r|}
\hline Point & $\mathbf{x}(\mathbf{m m})$ & $\mathbf{y}(\mathbf{m m})$ \\
\hline $\mathrm{m} 0$ & 0 & 0 \\
\hline $\mathrm{m} 1$ & 0 & 800 \\
\hline $\mathrm{m} 2$ & 0 & 1600 \\
\hline $\mathrm{m} 3$ & 700 & 1600 \\
\hline $\mathrm{m} 4$ & 1400 & 1600 \\
\hline $\mathrm{m} 5$ & 2100 & 1600 \\
\hline $\mathrm{m} 6$ & 2800 & 1600 \\
\hline $\mathrm{m} 7$ & 2800 & 800 \\
\hline $\mathrm{m} 8$ & 2800 & 0 \\
\hline $\mathrm{m} 9$ & 2100 & 0 \\
\hline $\mathrm{m} 10$ & 1400 & 0 \\
\hline $\mathrm{m} 11$ & 700 & 0 \\
\hline
\end{tabular}

Table 1: Coordinates of the reference points.

\subsection{Simulation}

First we show that our algorithm works by using a simulation with perfect silhouettes. We asked a person to stand still at each of the positions indicated in figure 1 when the V-shaped occluder was not present. We generated perfect foreground/background masks to be used as the silhouette images. Then we generated the traditional visual hull, where we saved the number of voxels and its centroid. The value of $T_{\text {cov }}$ is taken to be equal to 0.9 as our input silhouettes are perfect. With this threshold we won't add unwanted cells because the coverage has to be rather big. Depending on the quality of the calibration and the input silhouettes, this parameter might be changed for your own experiments.

To test our algorithm, we generated new fore- ground/background masks, but this time like if the Vshaped occluder were present. This means a varying number of occluded cameras for the 12 positions (table 2). In figure 4 an example of the silhouette images for position 10 is given. In figure 5 we see the $3 \mathrm{D}$ reconstruction from these 7 input masks. Although 4 out of 7 camera views are clearly occluded, our algorithm still succeeds to build a $3 \mathrm{D}$ reconstruction that looks very much like the person.

\begin{tabular}{c|c|c|c|c|c|c|c|} 
& $\mathrm{C} 1$ & $\mathrm{C} 2$ & $\mathrm{C} 3$ & $\mathrm{C} 4$ & $\mathrm{C} 5$ & $\mathrm{C6}$ & $\mathrm{C} 7$ \\
\hline $\mathrm{m} 0$ & 90 & 0 & 0 & 0 & 0 & 37 & 0 \\
\hline $\mathrm{m} 1$ & 10 & 99 & 0 & 0 & 0 & 0 & 0 \\
\hline $\mathrm{m} 2$ & 0 & 61 & 0 & 0 & 0 & 0 & 0 \\
\hline $\mathrm{m} 3$ & 0 & 0 & 0 & 93 & 0 & 0 & 0 \\
\hline $\mathrm{m} 4$ & 0 & 0 & 0 & 91 & 0 & 0 & 0 \\
\hline $\mathrm{m} 5$ & 0 & 0 & 82 & 38 & 0 & 0 & 0 \\
\hline $\mathrm{m} 6$ & 0 & 0 & 78 & 0 & 0 & 0 & 0 \\
\hline $\mathrm{m} 7$ & 0 & 0 & 86 & 0 & 0 & 0 & 0 \\
\hline $\mathrm{m} 8$ & 0 & 0 & 0 & 0 & 52 & 0 & 0 \\
\hline $\mathrm{m} 9$ & 0 & 0 & 0 & 0 & 85 & 0 & 5 \\
\hline $\mathrm{m} 10$ & 34 & 0 & 0 & 0 & 89 & 91 & 88 \\
\hline $\mathrm{m} 11$ & 94 & 0 & 0 & 0 & 0 & 92 & 95 \\
\hline
\end{tabular}

Table 2: Percentage of occlusion for all cameras on the 12 reference positions. $100 \%$ means fully occluded, $0 \%$ means no occlusion in that view. There is rather severe occlusion, even in multiple cameras for the same measuring point.

The input masks with the occluder present are used as input for our algorithm and the aim is to recover the whole person even though some cameras are occluded now. We compare both the number of voxels and the centroid for $(\mathrm{x}, \mathrm{y})$ and $(\mathrm{x}, \mathrm{y}, \mathrm{z})$ in figure 6 . We clearly see that our algorithm outperforms the tradi- 
tional algorithm and the euclidean distance between the centroid from our algorithm and the actual centroid is lower than $20 \mathrm{~mm}$ (the voxel size).

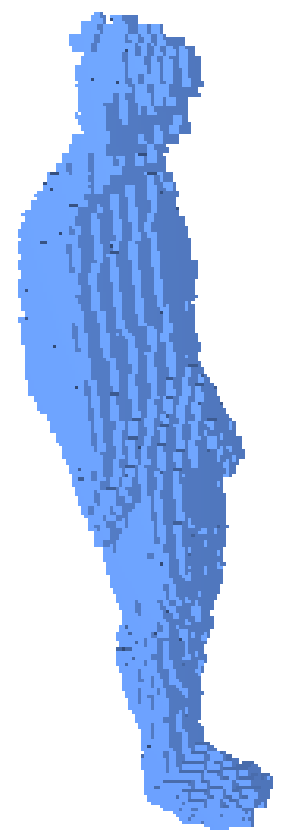

Figure 5: 3D reconstruction based on the input images of figure 4 . The complete person is reconstructed while only 4 out of 7 cameras could see the complete person. Voxel size is $2 \mathrm{~cm} \times 2 \mathrm{~cm} \times 2 \mathrm{~cm}$. The traditional algorithm only reconstructs a few voxels from the head of the person.

\subsection{Real world data}

In this section we will show that our proposed algorithm also has a lot of potential in the real world. For this we build an office environment in the multicamera setup we also used for simulations in the previous section of this paper (figure 3). The desktop environment is composed by four static objects: a V-shaped wall, a desk, a TV screen and a chair. In figure 7 we show the floorplan of this setup and the trajectory we calculate from the seven input video streams. The red dots represent the centroid of the person using the traditional visual hull algorithm while the blue dots represent the centroid calculated from the output of our proposed algorithm. As you can see the blue dots are much more represented than the red dots. Only in $36.9 \%$ of the frame sets, the traditional visual hull produces an output, while our algorithm produces a position for all frame sets.

Note that the produced result in figure 7 is less

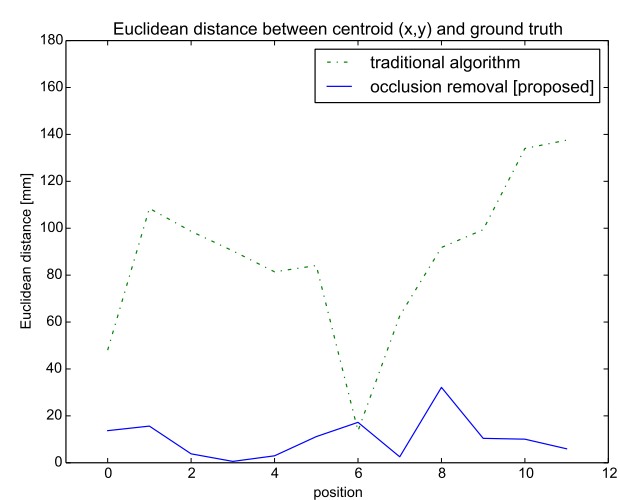

(a) Error centroid $(x, y)$

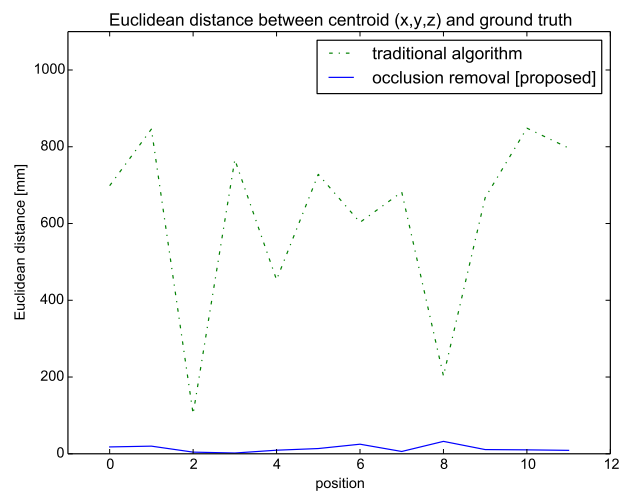

(b) Error centroid $(\mathrm{x}, \mathrm{y}, \mathrm{z})$

Figure 6: Visual representation of euclidean distance between the $(\mathrm{x}, \mathrm{y})$-coordinate (a) and between the $(\mathrm{x}, \mathrm{y}, \mathrm{z})$ coordinate (b) of the centroid. The error on the $\mathrm{z}$-coordinate is most significant since the traditional algorithm only reconstruct part of the head, bringing the centroid to the head, far away from the actual centroid. Projecting on the xy plane ignores the z-coordinate, but even there we see that our algorithm gives lower error rates. The mean absolute error MAE for (a) is $10.52 \mathrm{~mm}$ and $87.52 \mathrm{~mm}$ and for (b) $13.38 \mathrm{~mm}$ and $616.42 \mathrm{~mm}$ for our algorithm and the traditional algorithm respectively.

accurate than the results in the simulations. This is mainly due to imperfect foreground/background segmentation errors and calibration errors. In simulations we produced perfect silhouettes as input for our algorithm whereas in this realworld example we took the output of the foreground/background segmentation method SUBSENSE [St-Charles et al., 2014]. This method performs among the best in the CDnet 2014 Change Detection benchmark [Wang et al., 2014], but still shows significant errors on the produced silhouettes. 


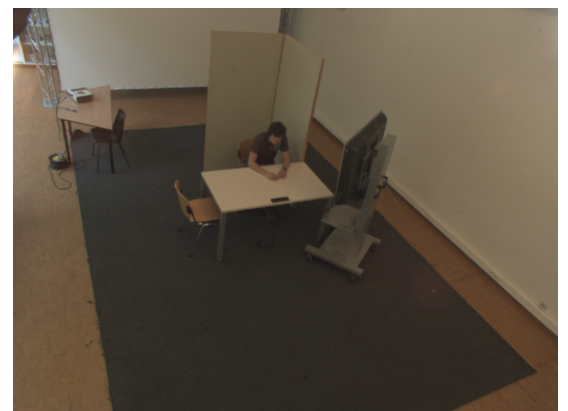

(a)

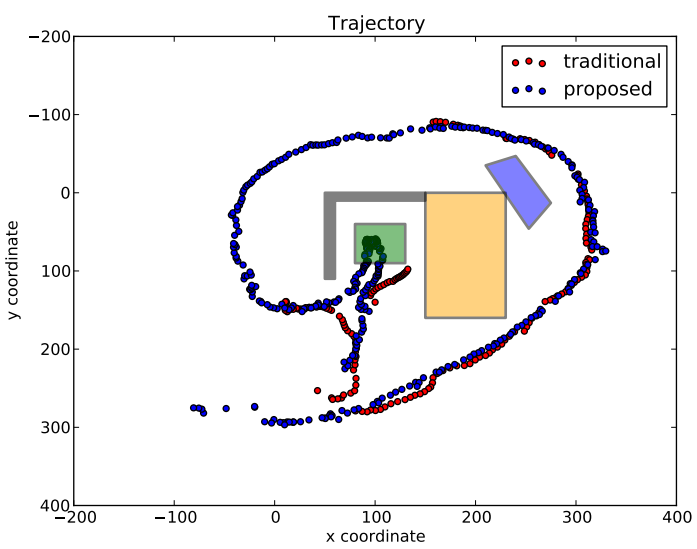

(b)

Figure 7: This figure shows the result of the trajectory of a person that enters the room in the bottom, walks to the table (yellow) and sits there on a chair (green), stands up from this chair and walks around his workplace. The blue dots represent our proposed algorithm and produce output for the complete trajectory (even with severe occlusion due to the table, TV screen (blue) and the wall (gray). The traditional visual hull algorithm only outputs positions for $36.9 \%$ of the frames. Visual inspection learns that the blue dots are much closer to the actual person than the red dots. The camera setup is the same as in figure 3 .

\section{Conclusion}

In this paper we proposed an algorithm to generate a 3D shape even if camera views are (partially or fully) occluded without the need for any prior knowledge of the scene (except for the camera calibration). We showed promising result in both simulations and real world examples which means it can have applications in real world environments where occlusion is a significant problem. In the absence of occlusion our algorithm can also be used because it also repairs holes in the 3D shape due to imperfect foreground/background segmentation because these holes can be treated as a form of occlusion.

The algorithm can handle both static and dynamic occlusion because it operates on a frame by frame basis without temporal information of the occluders. In future work this information could be integrated.

\section{REFERENCES}

Allied Vision Technologies. Manta G-046C. http: //www . alliedvisiontec.com/us/products/cameras/ gigabit-ethernet/manta/g-046bc.html. Accessed: 2014-09-14.

Guan, L., Sinha, S., Franco, J.-S., and Pollefeys, M. (2006). Visual hull construction in the presence of partial occlusion. In 3D Data Processing, Visualization, and Transmission, Third International Symposium on, pages 413-420. IEEE.

Laurentini, A. (1994). The visual hull concept for silhouette-based image understanding. Pattern Analysis and Machine Intelligence, IEEE Transactions on, 16(2):150-162.

Laurentini, A. (1997). How many 2 d silhouettes does it take to reconstruct a $3 \mathrm{~d}$ object? Computer Vision and Image Understanding, 67(1):81-87.

Laurentini, A. (1999). The visual hull of curved objects. In In Proceedings of ICCV'99, Corfu, pages 356-361.

Ober-Gecks, A., Haenel, M., Werner, T., and Henrich, D. (2014). Fast multi-camera reconstruction and surveillance with human tracking and optimized camera configurations. In ISR/Robotik 2014; 41st International Symposium on Robotics; Proceedings of, pages 1-8. VDE.

Slembrouck, M., Van Cauwelaert, D., Van Hamme, D., Van Haerenborgh, D., Van Hese, P., Veelaert, P., and Philips, W. (2014). Self-learning voxel-based multicamera occlusion maps for $3 \mathrm{~d}$ reconstruction. In 9 th International Joint Conference on Computer Vision, Imaging and Computer Graphics Theory and Applications (VISAPP-2014). SCITEPRESS.

St-Charles, P.-L., Bilodeau, G.-A., and Bergevin, R. (2014). Flexible background subtraction with self-balanced local sensitivity. In Proceedings of IEEE Workshop on Change Detection.

Stengel, D., Wiedemann, T., and Vogel-Heuser, B. (2012). Efficient $3 \mathrm{~d}$ voxel reconstruction of human shape within robotic work cells. In Mechatronics and $\mathrm{Au}$ tomation (ICMA), 2012 International Conference on, pages 1386-1392. IEEE.

Toth, C., O'Rourke, J., and Goodman, J. (2004). Handbook of Discrete and Computational Geometry, Second Edition. Discrete and Combinatorial Mathematics Series. Taylor \& Francis.

Wang, Y., Jodoin, P.-M., Porikli, F., Konrad, J., Benezeth, Y., and Ishwar, P. (2014). Cdnet 2014: An expanded change detection benchmark dataset. In Proceedings of the IEEE Conference on Computer Vision and Pattern Recognition Workshops, pages 387-394. 